\title{
A unique variant of spontaneous ovarian hyperstimulation syndrome: case report
}

\author{
Tanya Buckshee Rohatgi ${ }^{1}$, Kamal Buckshee ${ }^{2 *}$
}

\begin{abstract}
${ }^{1}$ Department of Reproductive Medicine and Assisted Reproductive Techniques (IVF), Max Medcentre TM and Max Super Speciality Hospital (Saket), Delhi, India and The Woman Clinic, Noida, Uttar Pradesh, India

${ }^{2}$ The woman Clinic, Noida, Uttar Pradesh, India
\end{abstract}

Received: 12 July 2017

Accepted: 05 August 2017

\author{
*Correspondence: \\ Dr. Kamal Buckshee, \\ E-mail: kamalbuckshee@gmail.com
}

Copyright: (c) the author(s), publisher and licensee Medip Academy. This is an open-access article distributed under the terms of the Creative Commons Attribution Non-Commercial License, which permits unrestricted non-commercial use, distribution, and reproduction in any medium, provided the original work is properly cited.

\begin{abstract}
Spontaneous ovarian hyperstimulation syndrome (SOHSS) is extremely rare in naturally conceived pregnancies and is potentially a life-threatening condition. Objective of present study was to highlight the development of new and atypical findings associated with SOHSS in a woman who conceived naturally with singleton pregnancy while taking inositols and metformin. A 35 years old lady was under pressure to conceive, so she presented to us for fertility consultation and further management. Being a case of polycystic ovary syndrome (PCOS) with irregular periods, hyperandrogenism and dyslipidemia with raised blood sugar level, she was started on inositols and metformin to aid fertility and to improve endocrine and metabolic parameters. To evaluate the status of ovaries and endometrium, ultrasound (US) was done which revealed moderate amount of free fluid in pelvis, thickened endometrium and mild enlargement of ovaries with unusually small and atypical follicles. Pregnancy test and beta-human chorionic gonadotropin $(\beta \mathrm{HCG})$ confirmed pregnancy. She was asymptomatic and was managed conservatively. Pregnancy progressed and at term, a healthy male baby was delivered. Awareness and suspicion of SOHSS in a PCOS case aided early diagnosis and timely management. Intake of type of pharmacological and non-pharmacological agents to be monitored for their impact on fertility, ovarian enlargement, severity of ovarian hyperstimulation syndrome (OHSS), clinical presentation, laboratory profile and outcome of pregnancy.
\end{abstract}

Keywords: Inositols, Ovarian hyperstimulation syndrome, Polycystic ovary syndrome

\section{INTRODUCTION}

The incidence of spontaneous OHSS in a naturally conceived pregnancy is a rare event. Scientific study on the pathophysiology of OHSS by Orvieto et al revealed increased ovarian volume due to the presence of multiple cysts and vascular hyper permeability, that resulted in the outflow of fluid from the intravascular space, with subsequent hypovolemia and hemoconcentration. ${ }^{1}$ Leener et al classified SOHSS in to Type 1: caused by FSH receptor mutations, Type 2: elevated human chorionic gonadotrophin (HCG) and Type 3: Hypothyroidism related. Primary risk factors for development of OHSS include: young age, PCOS, lean body weight, high dose of gonadotropins, elevated estradiol (E2) and HCG levels. ${ }^{2}$ Previous history of OHSS, molar pregnancy, multiple pregnancy, hypothyroidism and high basal anti mullerian hormone levels. ${ }^{3}$ In general SOHSS tends to present in late first trimester i.e. after 8-14 weeks of gestation. ${ }^{4}$ We report an unusual case of SOHSS at 4 weeks of gestation with mild enlargement of ovaries, atypical follicles (absence of multiple follicular cysts) in 
a PCOS woman with singleton gestation, who conceived naturally while taking inositols and metformin prior to conception. To the best of our knowledge this is probably the first case that is being reported and is of clinical significance.

\section{CASE REPORT}

A 35 years old lady, G2P0L0A1, was under pressure to conceive so she presented to our clinic on 11/6/2016 for advice and further management. Last menstrual period was on $14 / 5 / 16$.

\section{Menstrual history}

Menarche at 12 years. Since then, periods have been irregular and scanty, lasting for 2-3 days coming at an interval of 2-3-4-6 months, with weight gain and abnormal hair growth over lips, chin, face, arms, chest and abdomen. Last menstrual period (LMP) was on $14 / 5 / 16$.

\section{Obstetric history}

G2P0L0A1, missed abortion at 9 weeks. Past history: Investigated in 2006 and was diagnosed to have PCOS (Reports not available). She was advised Oral contraceptive pills (OCP) containing cyperoterone acetate, diet and life style management. She took treatment irregularly till 2007. After marriage (2007) she was reinvestigated for irregular periods and was advised weight reduction, OCP, metformin and life style management. Her weight decreased, periods became regular so she discontinued treatment because she wanted to plan pregnancy (2009). She tried to conceive by natural methods, homeopathic, ayurvedic and allopathic medicines from 2009 to 2015 . Induction of ovulation was attempted by clomiphene, letrazole and gonadotrophins but failed to conceive. Infertility work up of the husband revealed no abnormality and semen analysis was within normal limits, hysterosalpingography showed no abnormality, tubes were patent and ultrasound revealed polycystic ovaries (Figure 1, January 2015).

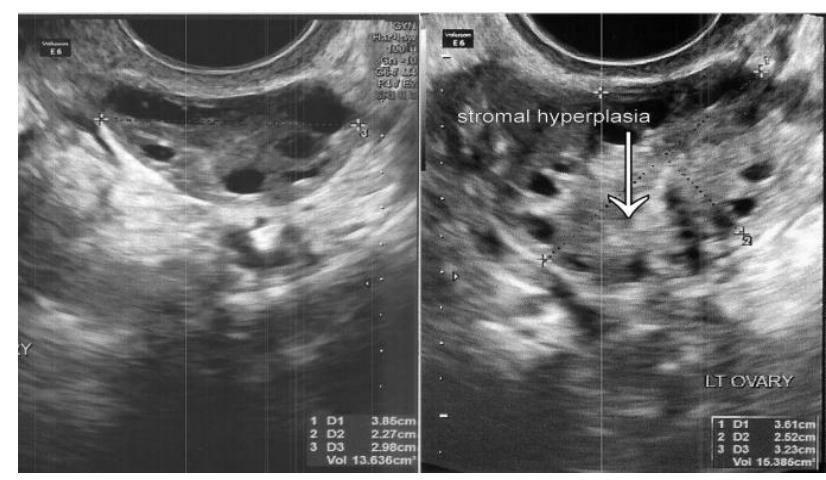

Figure 1: Polycystic ovary with stromal hyperplasia. Right ovary (RO) $13.63 \mathrm{~cm} 3$ and left ovary (LO) 15.38 cm3 (Ultrasound January 2015).
The couple came to us in May 2015 for infertility treatment. A diagnostic laparoscopy, hysteroscopy, and endometrial biopsy (histopathology and PCR for tuberculosis) was performed. Both tubes were patent and healthy with free spill on both sides, ovaries were enlarged and polycystic. Both ovaries were drilled and endometrium was in proliferative phase (June 2015). Since she had not conceived she went to consult an infertility specialist (January 2016), who on routine US detected missed abortion 9 weeks. This was her first conception. She had no symptoms of pregnancy and mistook amenorrhea as menstrual irregularity. Pregnancy was terminated and products revealed no chromosomal abnormality (23/01/2016). She again returned to us with amenorrhea of 2 months and 12 days. Investigations revealed $\beta \mathrm{HCG}<1.5 \mathrm{mIU} / \mathrm{ml}$, fasting blood sugar (FBS) was raised $107 \mathrm{mg} / \mathrm{dl}$, dyslipidemia, low vitamin $\mathrm{D}_{3} 25$ $\mathrm{OH}$, anticardiolipin and antiphospholipid antibodies were negative. She had withdrawal bleed on 30/03/2016. Folic acid, vitamin $\mathrm{D}_{3}$ 25-OH supplement, myo (550mgm) and d-chiro $(150 \mathrm{mgm})$ inositols were started along with metformin, being a case of PCOS with metabolic and endocrine abnormalities. She had spontaneous period on $14 / 05 / 2016$ after 45 days of withdrawal bleed (30/03/2016). She was asymptomatic, vitals were stable and weight $85 \mathrm{~kg}$, body mass index (BMI) $33.2 \mathrm{~kg} / \mathrm{m}^{2}$. Systemic examination revealed no abnormality. Routine US revealed moderate amount of free fluid in pelvis, thickened endometrium $14 \mathrm{~mm}$, right ovary (RO) $37 \times 33 \times 16 \mathrm{~mm}-10.44 \mathrm{ml}$ volume and left ovary (LO) 40x30x18mm-11.54ml volume. Moderate stromal hyperplasia in both ovaries with multiple atypical follicles and a single corpus luteum in LO. However, follicles appeared peculiar (shrivelled) and small (Figure 2). Whereas follicles in hyperstimulated ovaries are large, cystic and distended (Figure 3).

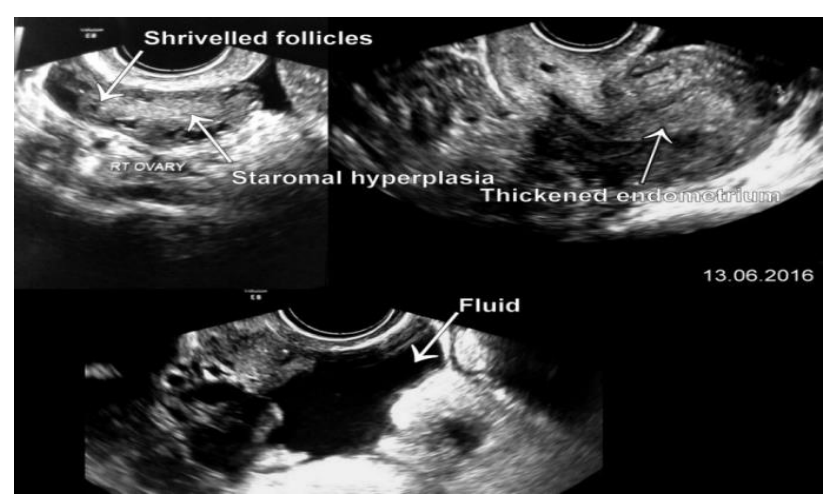

Figure 2: Moderate free fluid in pelvis, unusual follicles, stromal hyperplasia and thickened endometrium.

Pregnancy test was weakly positive. A provisional diagnosis was ectopic, spontaneous ovarian hyperstimulation syndrome with pregnancy (11/06/2016). A repeat US on 13/06/2016 reported same findings (Figure 2). Serum $\beta \mathrm{HCG}$, progesterone, liver, kidney, thyroid function, serum calcium, FBS, coagulation profile 
and complete blood count were done. Haemoglobin 12.10 $\mathrm{g} / \mathrm{l}$, hematocrit $37.3 \%$, platelet count $2,25,000 \mathrm{~mm}^{3}$, FBS $107 \mathrm{mg} / \mathrm{dL}$, serum $\beta$ HCG $85.92 \mathrm{mIU} / \mathrm{ml}$, progesterone $28.9 \mathrm{ng} / \mathrm{mL}$ and rest of the investigations were in normal range. Repeat serum $\beta \mathrm{HCG} 303.59 \mathrm{mIU} / \mathrm{ml}$ on 14/06/2016. A diagnosis of mild SOHSS mild ovarian enlargement unusually small follicles with absence of multiple follicular cysts with pregnancy was made. There was absence of hypovolemia, hemoconcentration and hematocrit was normal. Inositols were stopped because we were not aware of their effects in early pregnancy. However, we continued metformin to prevent recurrence of OHSS, to maintain endocrine and metabolic parameters. Luteal support was given. Fluids were restricted. Allowed to drink to quench thirst. Follow up US (30/06/2016) revealed resolution of free fluid (19 days), fetal pole with cardiac activity and $\beta \mathrm{HCG}$ of 37,569.49 $\mathrm{mIU} / \mathrm{ml}$. Pregnancy progressed, Level I and II ultrasounds were normal, except for an isolated choroid plexus cyst (CPC). Double marker and quadruple screen revealed low risk for down syndrome and neural defects with absence of CPC. At 34 weeks, she developed gestational diabetes, metformin dose was increased and insulin was started. She delivered a full term healthy 3.4 $\mathrm{kg}$ (male) by lower segment caesarean section for nonprogress of labour.

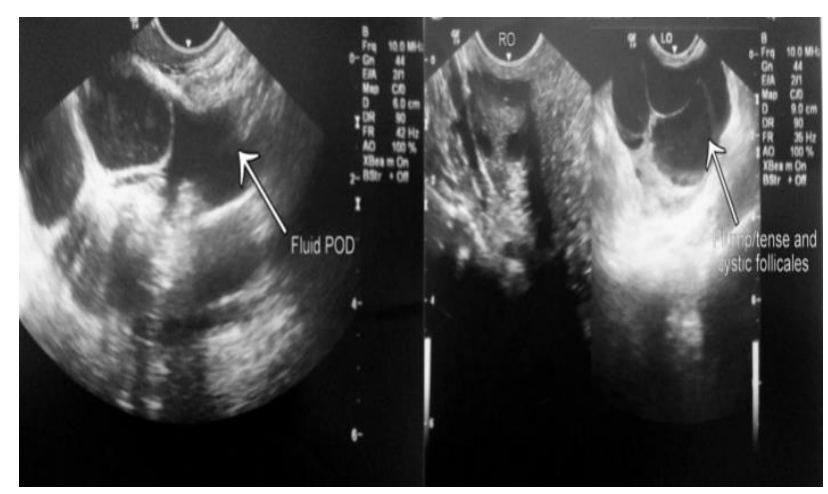

Figure 3: Large and cystic plump/tense follicles in OHSS.

\section{DISCUSSION}

Spontaneous OHSS is an extremely rare condition in a naturally conceived pregnancy. Our patient conceived spontaneously with singleton pregnancy, while taking combination of inositols and metformin which were started prior to conception. Being at risk of developing OHSS, raised sugar level, dyslipidemia and anxious to conceive metformin and combination of inositols were started to enhance fertility, improve endocrine and metabolic parameters, since she had failed to conceive while taking metformin alone. Benelli et al have demonstrated that combined therapy with myo-inositol (MI) and d-chiro-inosital (DCI) improves endocrine parameters and insulin resistance in PCOS young overweight women. It is a first line treatment in PCOS cases with hyperandrogenism, and menstrual dysfunction. ${ }^{5}$ Olatunbosun et al reported severe SOHSS associated with naturally conceived pregnancy in a woman with polycystic ovarian disease and resulted in live birth. ${ }^{6}$ However, our PCOS case developed mild OHSS, mild ovarian enlargement with unusually small and atypical follicles in contrast to huge ovarian enlargement with large plump and cystic follicles (Figure 3). Probably metformin and inositols acted on the ovaries and resulted in mild ovarian enlargement with atypical follicles (Figure 2). Perhaps, their functions also got altered which in turn resulted in diminished vascular permeability, VEGF, E2 levels and COX-2 expression. The net result was mild OHSS with no hypovolemia nor hemoconcentration. Animal experimental study indicates that metformin decreases the incidence of OHSS. But little is known about the possible effects and mechanism of action involved. ${ }^{7}$ Cochrane review reported that metformin increased clinical pregnancy rates and reduced OHSS risk with no effect on live birth rate. ${ }^{8}$

Smith et al have supported use of adjuvant metformin as a strategy for OHSS prevention starting at least 2 months before controlled ovarian stimulation. ${ }^{9}$ Turan et al demonstrated that although MI was similar to metformin in prevention of OHSS, but increase in the ovarian diameter was the smallest in the MI treated group. Vascular permeability, VEGF, E-2 levels and cox-2 expression were reduced in animals treated with $\mathrm{MI}$ and/or metformin and inositol was effective in preventing OHSS similar to metformin. ${ }^{10}$ Paplaeo et al observed lower E2 level on HCG day in the individuals receiving MI. Based on this finding they commented that MI reduces the risk of OHSS. However, no study has addressed this possibility as yet. ${ }^{11}$ Minozz et al reported that MI exerts its beneficial effects mainly at the ovarian level and acts on a number of ovarian functions. ${ }^{12}$ Extremely low haemoglobin concentration, $5.1 \mathrm{~g} / \mathrm{dl}$ has been reported along with retention of fluid in the extravascular compartment with absence of hemoconcentration. ${ }^{13}$ In present case there was moderate amount of fluid in the pelvic, mild ovarian enlargement, unusually small follicles with normal hematocrit. Spontaneous OHSS tends to present in late first trimester after 8-14 weeks of gestation. ${ }^{4}$ Whereas iatrogenic OHSS usually occurs earlier.

Present case presented at around 4 weeks of gestation. It probably occurs early, but diagnosis is missed, because patients usually report when they are symptomatic. Our patient was asymptomatic and presented early. Iatrogenic OHSS cases are detected early, because they are monitored frequently. Our patient was asymptomatic either due to mild OHSS, associated with mild ovarian enlargement and small follicles or because there was fluid only in the pelvis. Our case demonstrates that inositols and metformin had positive impact on fertility, modified severity of SOHSS, decreased ovarian and follicular enlargement and can present early. However, no clinical study has addressed the issue of early onset, beneficial effect of metformin and inositols on ovaries by 
decreasing its enlargement, preventing the formation of large multiple distended follicular cysts and reducing the severity of OHSS. Studies are needed to clarify possible effects and mechanism of action of combined therapy of metformin and inositols on ovaries and their functions. Thus, spontaneous OHSS is a separate entity, with varied presentation, its aetiology is multifactorial, pathophysiology and diagnostic criteria need clarification.

\section{CONCLUSION}

Present case provides new insights of SOHSS while taking metformin and inositols prior to conception. They improved fertility, modified severity of OHSS, prevented development of massive ovarian and follicular enlargement probably by acting at the ovarian level. Suspicion and awareness aided early diagnosis and timely management. In early spontaneous pregnancies, especially in PCOS cases both ovaries to be examined for prediction of hyperstimulation at earlier stages, if present meticulous follow up to be done.

Funding: No funding sources

Conflict of interest: None declared

Ethical approval: Not required

\section{REFERENCES}

1. Raoul O, Olga DS, Daniel L, Jigal H, Roy M, Yoram C. Interleukin-2 and SOCS-1 proteins involvement in the pathophysiology of severe ovarian hyperstimulation syndrome-a preliminary proof of concept. J Ovarian Res. 2014;7:106.

2. Leener AD, Montanelli L, Van Durme J, Chae H, Smits G, Vassart G, Costaglioola S. Presence and absence of follicle-stimulating hormone receptor mutations provide some insights into spontaneous ovarian hyperstimulation syndrome physiopathology. J Clin Endocrinol Metab. 2006;91:555-62.

3. Stolorz K, Nowosielski K, Włodarz IU, Sodowska P, Sodowski K. Ovarian hyperstimulation syndrome in spontaneous pregnancy. J Gynecol Res Obstet. 2016;2.1:005-9.

4. Demirel E, Turhan U, Ekmekci E, Subasioglu A, Kelekci S. Severe spontaneous ovarian hyperstimulation syndrome with cervical insufficiency: a case report. JFIV Reprod Med Genet. 2016;4:181.
5. Benelli E, Ghianda SD, Cosmo CD, Tonacchera M. Clinical study A Combined therapy with myoinositol and d-chiro-inositol improves endocrine parameters and insulin resistance in PCOS young overweight women. Int $\mathrm{J}$ Endocrinol. 2016;3204083:1-5.

6. Olatunbosun OA, Gilliland B, Brydon LA, Chizen DR, Pierson RA. Spontaneous OHSS in four consecutive pregnancies. Clin Exp Obstet Gynecol. 1996;23:127-32.

7. Evelin ME, Ramiro Q, Carlos C, María VB, Gastón RV, Dante AP et al. Metformin decreases the incidence of ovarian hyperstimulation syndrome: an experimental study. J Ovarian Res 2013;6:62.

8. Tso LO, Costello MF, Albuquerque LE, Andriolo RB, Freitas V. Metformin treatment before and during IVF or ICSI in women with polycystic ovary syndrome. Cochrane Database Syst Rev 2014;11:CD006105.

9. Smith V, Osianlis T, Vollenhoven B. Prevention of ovarian hyperstimulation syndrome: a review. Obstet Gynecol Int. 2015;2015:1-10.

10. Turan GA, Eskicioglu F, Sivrikoz ON, Cengiz H, Adakan S, Gur EB et al. Myo-inositol is a promising treatment for the prevention of ovarian hyperstimulation syndrome (OHSS): an animal study. Arch Gynecol Obstet. 2015;292:1163-71.

11. Papaleo E, Unfer V, Baillargeon JP, Fusi F, Occhi F, Santis LD. Myo-inositol may improve oocyte quality in intracytoplasmic sperm injection cycles. A prospective, controlled, randomized trial. Fertil Steril. 2009;91(5):1750-4.

12. Minnozi M, Costetino D, Gauraldi C, Unifer V. The effect of combination therapy with myo-inositol and combined oral contraceptive pill versus a combined oral contraceptive pill alone on metabolic, endocrine and clinical parameters in polycystic ovary syndrome. Gynecologic Endocrinol 2011;27(11):920-4.

13. Sharafeldin SMA, ELawad BE. Ovarian hyperstimulation syndrome in a 35-year old woman: A case report. Global Sci Res J. 2016;4(1):075-7.

Cite this article as: Rohatgi TB, Buckshee K. A unique variant of spontaneous ovarian hyperstimulation syndrome: case report. Int J Reprod Contracept Obstet Gynecol 2017;6:4170-3. 